\title{
On the Study of Network Coded AF Transmission Protocol for Wireless Multiple Access Channels
}

\author{
Zhiguo Ding, Member, IEEE, T. Ratnarajah, Senior Member, IEEE and Kin K. Leung, Fellow, IEEE
}

\begin{abstract}
In this paper, the performance of the network coded amplify-forward cooperative protocol is studied. The use of network coding can suppress the bandwidth resource consumed by relay transmission, and hence increase the spectral efficiency of cooperative diversity. A distributed strategy of relay selection is applied to the cooperative scheme, which can reduce system overhead and also facilitate the development of the explicit expressions of information metrics, such as outage probability and ergodic capacity. Both analytical and numerical results demonstrate that the proposed protocol can achieve large ergodic capacity and full diversity gain simultaneously.
\end{abstract}

Index Terms-cooperative diversity, network coding, multiple access channels, ergodic and outage capacities.

\section{INTRODUCTION}

C OOPERATIVE transmission offers a new dimension to mitigate the detrimental effects of multi-path fading by exploiting signals transmitted through direct and relay paths [1]-[3]. However, such relay transmission consumes extra bandwidth resource, which implies that the use of cooperative diversity typically results in the loss of system throughput. On the other hand, network coding has been independently developed in the context of wired communications and shown with the superior capability to increase system throughput [4], [5]. Hence it is nature to study the combination of network coding and cooperative diversity.

Network coded cooperative diversity has been previously studied in [6], where traditional network coding in [4] was applied. The idea of applying physical layer network coding [5] to cooperative multiple access channels (MAC) has been briefly discussed in our previous work [7] without analytical results. The aim of this correspondence is to provide a better understanding for such network coded cooperative MAC. A distributed strategy of relay selection is first applied to the proposed transmission protocol, which not only reduces the system overhead, but also makes the explicit analytical results feasible. Then the system robustness, in terms of outage

Manuscript received May 21, 2007; revised December 11, 2007 and April 9, 2008; accepted April 14, 2008. The associate editor coordinating the review of this letter and approving it for publication was Dr. A. Stefanov.

Z. Ding is with with the Department of Communication Systems of Lancaster University, Lancaster, LA1 4WA, UK and his work was supported by the UK EPSRC under grant number EP/F062079/1. (e-mail: z.ding@lancaster.ac.uk).

T. Ratnarajah is with ECIT, Queen's University of Belfast, Belfast, BT3 9DT, UK and his work was supported by the UK EPSRC under grant number EP/C004132/1.

K. K. Leung is with the Department of Electrical Engineering, Imperial College, London, SW7 2BT, UK and his work was supported by US Army Research laboratory and the UK Ministry of Defence and was accomplished under Agreement Number W911NF-06-3-0001.

Digital Object Identifier 10.1109/TWC.2008.070510. probability and diversity gain, is evaluated for the proposed scheme, which shows its ability to achieve the full diversity gain. Furthermore, the upper and lower bounds are developed for the achievable ergodic capacity, which demonstrates that the proposed scheme can achieve larger ergodic capacity than existing transmission schemes. Such balanced performance is due to the use of network coding, where one relay transmission can serve more than one source node simultaneously. As a result, the bandwidth resource consumed by relay transmission is reduced and the spectral efficiency of cooperative transmission is significantly improved, particularly in terms of ergodic capacity.

\section{Protocol Description And DATA Model}

Consider a communication scenario where $M$ sources transmit data to a common destination with the help of $L$ relays, which is an important building block for wireless communications. Time division duplex is applied here due to its simplicity, and the spectral efficiency of the developed protocol can be further improved by using more advanced multiple access techniques.

At the first time slot, all sources broadcast their messages simultaneously. Hence at this time slot, the destination receives $y_{D 1}=\sum_{m=1}^{M} h_{m D} s_{m}+n_{1}$, where $s_{m}$ is the message transmitted from the $m$ th source, $n_{1}$ is the additive Gaussian noise at the destination and $h_{m D}$ is the coefficient for the channel between the $m$ th source and the destination. In this paper, all wireless channels are assumed to be independent identical Raleigh fading. At the same time, each relay receives

$$
y_{R_{n}}=\sum_{m=1}^{M} h_{m R_{n}} s_{m}+n_{R_{n}}, \quad n \in\{1, \ldots, L\} .
$$

So after this first transmission, all relays received a mixture of the $M$ transmitted messages. The key idea of the proposed protocol is to introduce the idea of network coding into cooperative networks, where one relay transmission can help more than one source simultaneously.

Due to the dynamic nature of radio propagation, the connection of one relay with the destination and sources varies, which is critical to the system performance. Assume that $M-1$ relays have been selected to participate into cooperative, where the details for relay selection will be discussed at the end of this section. The amplify-forward strategy is used here for relay transmission. During the next $M-1$ time slots, the selected relays will take their turns to forward the mixture to the destination,

$$
y_{D(l+1)}=h_{R_{l} D} y \hat{R}_{l}+n_{l+1}, \quad l=1, \ldots, M-1,
$$


where $\hat{y}_{l}=y_{R_{l}} / \beta_{l}, \beta_{l}=\sqrt{\sum_{m=1}^{M}\left|h_{m R_{l}}\right|^{2}+1 / \rho}$ and $\rho$ is denoted as signal-to-noise ratio (SNR). Note that $\beta_{0}$ is defined as $\beta_{0}=\sqrt{\sum_{m=1}^{M}\left|h_{m D}\right|^{2}}$. It is assumed that the number of relays is larger than $(M-1)$.

So after $M$ time slots, the observations at the destination can be expressed as

$$
\mathbf{y}=\mathbf{D H s}+\mathbf{n},
$$

where $\mathbf{y}=\left[\begin{array}{lll}y_{D 1} & \cdots & y_{D M}\end{array}\right]^{T}, \mathbf{s}=\left[\begin{array}{lll}s_{1} & \cdots & s_{M}\end{array}\right]^{T}, \mathbf{D}=$ $\operatorname{diag}\left\{\beta_{0}, h_{R_{1} D}, \ldots, h_{R_{M-1} D}\right\}$,

$$
\begin{gathered}
\mathbf{H}=\left[\begin{array}{ccc}
h_{1 D} / \beta_{0} & \cdots & h_{M D} / \beta_{0} \\
h_{1 R_{1}} / \beta_{1} & \cdots & h_{M R_{1}} / \beta_{1} \\
\vdots & \vdots & \vdots \\
h_{1 R_{M-1}} / \beta_{M-1} & \cdots & h_{M R_{M-1}} / \beta_{M-1}
\end{array}\right] \\
\text { and } \mathbf{n}=\left[\begin{array}{c}
n_{1} \\
n_{2}+h_{R_{1} D} n_{R_{1}} / \beta_{1} \\
\vdots \\
n_{M}+h_{R_{M-1} D} n_{R_{M-1}} / \beta_{M-1}
\end{array}\right] .
\end{gathered}
$$

Note that the matrix $\mathbf{H}$ is not a regular Gaussian random matrix. Each of its row is normalized, and more importantly, the use of different relay selection strategies also has the impact on the distribution of $\mathbf{H}$.

The sum-rate achieved by the proposed transmission protocol can be written as

$$
\begin{array}{r}
\mathcal{I}=\frac{1}{M} \log \operatorname{det}\left\{\mathbf{I}_{M}+\rho \mathbf{D} \mathbf{H} \mathbf{H}^{H} \mathbf{D}^{H} \mathbf{C}^{-1}\right\} \\
\approx \log \rho+\frac{1}{M} \log \operatorname{det}\left\{\mathbf{D}^{H} \mathbf{D} \mathbf{C}^{-1}\right\}+\frac{1}{M} \log \operatorname{det}\left\{\mathbf{H} \mathbf{H}^{H}\right\}
\end{array}
$$

where the high SNR assumption is applied, $\mathbf{C}=\operatorname{diag}\{1,1+$ $\left.\left|h_{R_{1} D}\right|^{2} / \beta_{1}^{2}, \cdots, 1+\left|h_{R_{M-1} D}\right|^{2} / \beta_{M-1}^{2}\right\}$, and

$$
\log \operatorname{det}\left\{\mathbf{D}^{H} \mathbf{D} \mathbf{C}^{-1}\right\}=\frac{1}{M} \log \beta_{0}^{2} \prod_{l=1}^{M-1} \frac{\left|h_{R_{l} D}\right|^{2} \beta_{l}^{2}}{\left|h_{R_{l} D}\right|^{2}+\beta_{l}^{2}} .
$$

As discussed previously, the choice of the selected relays is crucial to the system performance. With the assumption of full channel state information (CSI), a centralized strategy can be easily developed by enumerating all possible choices of relays and choosing the $M-1$ ones which give the largest value of the sum rate ${ }^{1}$ in (3). Although such an optimal strategy shall maximize the ergodic sum-rate, it could cause too much system overhead, which motivates the following distributed strategy.

\section{A. Distributed Relay Selection Strategy}

Instead of the full CSI assumption, it is reasonable to assume that each relay has the knowledge to its incoming and outgoing channel information. Such local CSI can be obtained by asking the $M$ sources and the destination to broadcast pilot symbols, which consumes $(M+1)$ extra time slots. Ideally each relay should make a decision whether to participate into cooperation only based on its local CSI. And the key question

\footnotetext{
${ }^{1}$ Although the system robustness is not used as the criterion for relay selection, the proposed two relay selection strategies can provide the full diversity gain as shown in the next section.
}

is how such distributed decisions can maximize the overall system throughput in (3).

Consider that the expression of the sum-rate can be approximated as (4) at high SNR. The factor $\log \operatorname{det}\left\{\mathbf{D}^{H} \mathbf{D} \mathbf{C}^{-1}\right\}$ plays an important role for the sum-rate, and a good relay selection strategy should be able to yield a large value for the following variable

$$
\log \prod_{l=1}^{M-1} \frac{\left|h_{R_{l} D}\right|^{2} \beta_{l}^{2}}{\left|h_{R_{l} D}\right|^{2}+\beta_{l}^{2}}=\sum_{l=1}^{M-1} \log \frac{\left|h_{R_{l} D}\right|^{2} \beta_{l}^{2}}{\left|h_{R_{l} D}\right|^{2}+\beta_{l}^{2}} .
$$

Hence the value of $\frac{\left|h_{R_{l} D}\right|^{2} \beta_{l}^{2}}{\left|h_{R_{l} D}\right|^{2}+\beta_{l}^{2}}$ can be used as the criterion for each relay to make its decision whether to be involved in cooperation. In specific, a distributed strategy of relay selection to achieve a large value of the sum-rate can be easily implemented as the following [2]. Each relay will calculate its carrier sensing backoff time inversely proportional to the value $\frac{\left|h_{R_{l} D}\right|^{2} \beta_{l}^{2}}{\left|h_{R_{l} D}\right|^{2}+\beta_{l}^{2}}$ which is a function of its local CSI. Then during the $M-1$ time slots following the initial source broadcasting, the $M-1$ relays with the largest value of $\frac{\left|h_{R_{l} D}\right|^{2} \beta_{l}^{2}}{\left|h_{R_{l} D}\right|^{2}+\beta_{l}^{2}}$ can be selected for relay transmission, which ensures to obtain a large value of the system throughput. In the rest of this paper, the use of such a distributed relay selection strategy will be assumed since it can significantly simplify the development of explicit analytical results.

\section{B. Optimality of the Source Number $M$}

A nature question for the proposed protocol is how many sources and relays should be invited for network coded cooperative transmission. Intuition is that the more relays we have, the better quality relay we can find and hence the better performance we can obtain. However, the relationship between the number of sources and the system performance is not that straightforward. On one hand, with more sources participating, one relay transmission can serve more sources due to the use of network coding. On the other hand, a large number of $M$ makes it difficult to find a relay which can have good connections with the multiple sources simultaneously. This resembles the so-called "channel hardening" effect in MIMO systems where the increase of transceiver antennas could reduce the obtainable multi-user diversity. To answer this question, the following conjecture is provided.

Conjecture 1: The sum-rate achieved by the proposed transmission protocol can be maximized where there are only two sources participating cooperation.

We are yet to find a formal proof of this, although our simulations indicate that it is the case. In Fig. 1, the sum rate is shown as a function of the number of sources participating in cooperation. The number of relays is fixed as $L=10$ and only $M-1$ relays will be opportunistically used. As can be seen from Fig. 1, the ergodic sum rate $\mathcal{E}\{I\}$ is always inversely proportional to $M$ for all SNR. In practice, the fact that $M=2$ is optimal is beneficial since the system complexity can be reduced significantly.

\section{Outage Probability and Diversity Gain}

As discussed in the previous section, the optimal number of sources to participate into transmission is $M=2$, and 
hence in the rest of this paper, the scenario with $M=2$ sources and $L$ relays will be focused. The aim of this section is to evaluate the system robustness achieved by the proposed protocol, where two information theoretic metrics will be used, outage probability and diversity gain respectively.

Note that the addressed communication scenario can be viewed as one type of multiple access channels. And following the same definition in [8], [9], the outage event can be defined as

$$
\mathcal{O} \triangleq \bigcup_{\mathcal{A}} \mathcal{O}_{\mathcal{A}}
$$

where the union is taken over all possible subsets $\mathcal{A} \subseteq\{1,2\}$, and $\mathcal{O}_{\mathcal{A}}$ can be defined as

$$
\mathcal{O}_{\mathcal{A}} \triangleq\left\{\mathcal{I}\left(\mathbf{s}_{\mathcal{A}} ; \mathbf{y} \mid \mathbf{s}_{\mathcal{A}^{c}}, \mathbf{H}=H, \mathbf{D}=D\right) \leq \sum_{i \in \mathcal{A}} R_{i}\right\} .
$$

Furthermore, define $|\mathcal{A}|$ as the number of users in $\mathcal{A}$. Note that the symmetric system is of interest in this paper, which means $|\mathcal{A}| R=\sum_{i \in \mathcal{A}} R_{i}$. Since only the two-user scenario is considered here, the mutual information can be written as

$$
\mathcal{I}_{\mathcal{A}_{i}}=\log \left[1+\rho\left(\left|h_{i D}\right|^{2}+\frac{\left|h_{R D}\right|^{2}\left|h_{i R}\right|^{2}}{\left|h_{i R}\right|^{2}+\left|h_{R D}\right|^{2}}\right)\right], i \in\{1,2\},
$$

$$
\text { and } \mathcal{I}_{\mathcal{A}_{3}}=\log \operatorname{det}\left\{\mathbf{I}+\rho \mathbf{D H} \mathbf{H}^{H} \mathbf{D}^{H} \mathbf{C}^{-1}\right\},
$$

where $\mathcal{I}_{\mathcal{A}_{n}}=\mathcal{I}\left(\mathbf{s}_{\mathcal{A}_{n}} ; \mathbf{y} \mid \mathbf{s}_{\mathcal{A}_{n}^{c}}, \mathbf{H}=H, \mathbf{D}=D\right)$. In this paper, we use the special symbol $\doteq$ to denote exponential equality [8], i.e., $f(\rho) \doteq \rho^{n}$ to denote $\lim _{\rho \rightarrow \infty} \frac{\log f(\rho)}{\log \rho}=n$. The following theorem provides the diversity gain and the highSNR approximation for the outage probability achieved by the proposed transmission protocol.

Theorem 2: Assume that all CSI are i.i.d Raleigh fading. For the scenario with two sources and $L$ relays, the outage probability of the proposed network coded transmission protocol can be approximated at high SNR as

$$
P(\mathcal{O}) \doteq \frac{1}{\rho^{L+1}}
$$

Proof: The proof for this theorem can be accomplished in two steps. For the first step, it will be proved that $P\left(\mathcal{I}_{\mathcal{A}_{3}} \leq\right.$

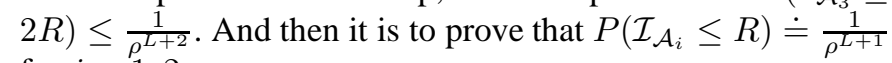
for $i=1,2$.

Define $\gamma=\frac{\left|h_{1, R}\right|^{2}+\left|h_{2, R}\right|^{2}}{\left|h_{1, R}\right|^{2}+\left|h_{2, R}\right|^{2}+\left|h_{R, D}\right|^{2}}$. So firstly rewrite the expression of $\mathcal{I}_{\mathcal{A}_{3} \text { as }}^{\left|h_{1, R}\right|^{2}}$

Define a complex variable as $w=h_{1, D} h_{2, R}-h_{2, D} h_{1, R}$, and further denote its real and imaginary parts as $w=a+j b$. Then we can obtain

$$
\begin{aligned}
\left|h_{1, D}\right|^{2}\left|h_{2, R}\right|^{2}+\left|h_{2, D}\right|^{2}\left|h_{1, R}\right|^{2}- & 2 \mathcal{R}\left\{h_{1, D}^{*} h_{2, D} h_{1, R} h_{2, R}^{*}\right\} \\
& =\mathcal{R}\left\{w w^{*}\right\}=a^{2} \geq 0 .
\end{aligned}
$$

By using such a fact, the mutual information can be lower bounded as

$$
\begin{aligned}
\mathcal{I}_{\mathcal{A}_{3}} \geq & \log \left[1+\rho\left(\left|h_{1, D}\right|^{2}+\left|h_{2, D}\right|^{2}\right)\right. \\
& \left.\rho \frac{\left|h_{1, R}\right|^{2}+\left|h_{2, R}\right|^{2}}{\left|h_{1, R}\right|^{2}+\left|h_{2, R}\right|^{2}+\left|h_{R, D}\right|^{2}}\left|h_{R, D}\right|^{2}\right] .
\end{aligned}
$$

Using this lower bound of the mutual information, the outage probability can be upper bounded as

$$
P\left(I_{\mathcal{A}_{3}}<2 R\right) \leq P\left(x+y<\frac{2^{2 R}-1}{\rho}\right),
$$

where $x=\beta_{0}^{2}$ and $y=\frac{\left|h_{R D}\right|^{2} \beta_{1}^{2}}{\left|h_{R D}\right|^{2}+\beta_{1}^{2}}$. Since $x$ is a sum of four i.i.d. Gaussian variables, its distribution is the Chi-square function with 4 degree of freedom, $f_{x}(x)=x e^{-x}$. The density function of $y$ is more complicated as it is a function of an exponentially distributed variable $y_{1}=\left|h_{R D}\right|^{2}$ with $f_{y_{1}}=$ $e^{-y_{1}}$, and another Chi-square distributed variable $y_{2}=\beta_{1}$ with $f_{y_{2}}\left(y_{2}\right)=y_{2} e^{-y_{2}}$.

Given $y=\frac{y_{1} y_{2}}{y_{1}+y_{2}}$, the density function of the variable $y$ can be found as

$$
\begin{aligned}
P(y) & =\int_{y}^{\infty} y_{2} e^{-y_{2}}\left[1-e^{-\frac{y_{2} y}{y_{2}-y}}\right] d y_{2}+\int_{0}^{y} y_{2} e^{-y_{2}} d y_{2} \\
& =1-2 y^{2} e^{-2 y}\left[\mathbf{K}_{-1}(2 y)+\mathbf{K}_{-2}(2 y)\right]
\end{aligned}
$$

where $\mathbf{K}_{1}(x)$ is the modified bessel function of the second kind with first order. After applying relay selection, the relay with the largest value of the criterion $\frac{\left|h_{R D}\right|^{2} \beta_{1}^{2}}{\left|h_{R D}\right|^{2}+\beta_{1}^{2}}$ will be chosen. Hence the use of the relay selection strategy changes the density function of the variable $y$ as

$$
P(y)=\left\{1-2 y^{2} e^{-2 y}\left[\mathbf{K}_{-1}(2 y)+\mathbf{K}_{-2}(2 y)\right]\right\}^{L} .
$$
Define $\alpha=\frac{2^{2 R}-1}{\rho}$. Now the outage probability can be finally
upper bounded as

For large SNR, we have $\alpha \rightarrow 0$. And for small value of $x$, the bessel functions can be approximated as $\mathbf{K}_{2}(x) \approx \frac{2}{x^{2}}$ and $\mathbf{K}_{1}(x) \approx \frac{1}{x}$. Further utilizing the fact that $\mathbf{K}_{-n}(x)=\mathbf{K}_{n}(x)$, the following approximation can be obtained as

$$
\begin{aligned}
P\left(I_{\mathcal{A}_{3}}<2 R\right) & \leq \int_{0}^{\alpha} x\left\{1-e^{-2(\alpha-x)}\right\}^{L} d x \\
& \approx \frac{2^{L} \alpha^{L+2}}{(L+1)(L+2)} \doteq \frac{1}{\rho^{L+2}} .
\end{aligned}
$$

which completes the first step of this proof.

On the other hand, it is obvious that $P\left(I_{\mathcal{A}_{1}}<R\right)=$ $P\left(I_{\mathcal{A}_{2}}<R\right)$ due to the system symmetry. So in the following, we only focus on the outage probability $P\left(I_{\mathcal{A}_{1}}<R\right)$, which can be shown that

$$
\mathcal{I}_{L, \mathcal{A}_{1}}=\log \left[1+\rho\left(\left|h_{1 D}\right|^{2}+\frac{\left|h_{R D}\right|^{2}\left|h_{1 R}\right|^{2}}{\left|h_{1 R}\right|^{2}+\left|h_{R D}\right|^{2}}\right)\right](.
$$

Define $z=\frac{z_{1} z_{2}}{z_{1}+z_{2}}$ where $z_{1}=\left|h_{R D}\right|^{2}$ and $z_{2}=\left|h_{1 R}\right|^{2}$. Since both $z_{1}$ and $z_{2}$ are i.i.d. exponentially distributed, the PDF of $z$ can be shown as

$$
\begin{aligned}
P(z) & =\int_{z}^{\infty} e^{-z_{1}}\left[1-e^{-\frac{z_{1} z}{z_{1}-z}}\right] d z_{1}+\int_{0}^{z} e^{-z_{1}} d z_{1} \\
& =1-\int_{z}^{\infty} e^{-z_{1}} e^{-\frac{y z}{z_{1}-z}} d z_{1}=1-2 z e^{-2 z} \mathbf{K}_{1}(2 z) .
\end{aligned}
$$

After applying relay selection, the outage probability is

$$
\begin{array}{r}
P\left(\mathcal{I}_{L, \mathcal{A}_{1}} \leq R\right)=P\left(x+z<2^{R}-1\right) \\
=\int_{0}^{\alpha} e^{-x}\left[1-2(\alpha-x) e^{-2(\alpha-x)} \mathbf{K}_{1}(2 \alpha-2 x)\right]^{L} d x .
\end{array}
$$




$$
\begin{aligned}
\mathcal{I}_{\mathcal{A}_{3}}= & \log \left[1+\rho \gamma\left|h_{R, D}\right|^{2}+\rho\left(\left|h_{1, D}\right|^{2}+\left|h_{2, D}\right|^{2}\right)+\frac{\rho^{2}\left|h_{R, D}\right|^{2}}{\left|h_{1, R}\right|^{2}+\left|h_{2, R}\right|^{2}+\left|h_{R, D}\right|^{2}}\right. \\
& \left.\times\left(\left|h_{1, D}\right|^{2}\left|h_{2, R}\right|^{2}+\left|h_{2, D}\right|^{2}\left|h_{1, R}\right|^{2}-2 \mathcal{R}\left\{h_{1, D}^{*} h_{2, D} h_{1, R} h_{2, R}^{*}\right\}\right)\right] . \\
P\left(I_{\mathcal{A}_{3}}<2 R\right) \leq & \int_{0}^{\alpha} x e^{-x}\left\{1-2(\alpha-x)^{2} e^{-2(\alpha-x)}\left[\mathbf{K}_{-1}(2 \alpha-2 x)+\mathbf{K}_{-2}(2 \alpha-2 x)\right]\right\}^{L} d x .
\end{aligned}
$$

With the high SNR assumption, the bessel function can be approximated as $\mathbf{K}_{1}(x) \approx \frac{1}{x}$, which results

$$
\begin{aligned}
P\left(\mathcal{I}_{L, \mathcal{A}_{1}}\right) & \approx \int_{0}^{\alpha} e^{-x}\left[1-e^{-2(\alpha-x)}\right]^{N} d x \\
& \approx \int_{0}^{\alpha}[2(\alpha-x)]^{N} d x=2^{L} \frac{\alpha^{L+1}}{L+1} \doteq \frac{1}{\rho^{L+1}}
\end{aligned}
$$

The overall outage probability $P(\mathcal{O})$ shall be bounded as the following

$$
\frac{1}{\rho^{L+1}} \doteq P\left(\mathcal{O}_{\mathcal{A}}\right) \leq P(\mathcal{O}) \leq \sum_{\mathcal{A}} P\left(\mathcal{O}_{\mathcal{A}}\right) \doteq \frac{1}{\rho^{L+1}}
$$

And the proof for Theorem 2 is completed.

The key message delivered by Theorem 2 is that the full diversity gain $L+1$ can be achieved by the proposed transmission protocol, which is also achievable by many existing cooperative protocols in [2], [9]. Then the question is whether the proposed protocol can offer any other benefits while maintaining the full diversity property, which is answered in the next section.

\section{EROGOTIC CAPACITY}

Definition 1: Ergodic capacity is the long-term data rate that a system can support, i.e.,

$$
\mathcal{C}_{e}=\int_{0}^{\infty} \mathcal{I} f_{\mathcal{I}}(\mathcal{I}) d \mathcal{I}
$$

where $f_{\mathcal{I}}(\cdot)$ is the probability density function (PDF) of the mutual information $\mathcal{I}$.

In the following theorem the ergodic capacity based on sum rate will be provided for the proposed protocol.

Theorem 3: Assume all channels are i.i.d. Raleigh fading. The ergodic capacity achieved by the proposed network coded cooperative transmission protocol can be bounded as

$$
\begin{aligned}
& \mathcal{E}\left\{\mathcal{I}_{D}\right\}+\frac{1}{2}\left(\sum_{k=1}^{L} C_{L}^{k}(-1)^{k} \log 4 k\right) \leq \mathcal{E}\{\mathcal{I}\} \\
& \leq \mathcal{E}\left\{\mathcal{I}_{D}\right\}+\frac{1}{2}\left(\sum_{k=1}^{L} C_{L}^{k}(-1)^{k} \log k\right) .
\end{aligned}
$$

where $\mathcal{E}\left\{\mathcal{I}_{D}\right\} \approx \log \rho-\mathbf{C} \log e$ is the ergodic capacity achieved by direct transmission.

Proof: Following the previous discussion, the ergodic capacity achieved by the proposed transmission protocol can be approximated at high SNR as

$$
\begin{aligned}
\mathcal{E}\{\mathcal{I}\} \approx \log \rho+\frac{1}{2} \mathcal{E} & \left\{\log \operatorname{det}\left[\mathbf{D}^{H} \mathbf{D} \mathbf{C}^{-1}\right]\right\} \\
+ & \frac{1}{2} \mathcal{E}\left\{\log \operatorname{det}\left[\mathbf{H} \mathbf{H}^{H}\right]\right\}
\end{aligned}
$$

which is a function of two variables, $\log \operatorname{det}\left[\mathbf{D}^{H} \mathbf{D} \mathbf{C}^{-1}\right]$ and $\log \operatorname{det}\left[\mathbf{H H}^{H}\right]$.

The exact expression for the ergodic capacity will be difficult, and hence we will be focusing on developing the upper bound and lower bound of the capacity $\mathcal{E}\{\mathcal{I}\}$, which can be accomplished in two steps. First the expectation of $\frac{1}{2} \mathcal{E}\left\{\log \operatorname{det}\left[\mathbf{D}^{H} \mathbf{D} \mathbf{C}^{-1}\right]\right\}$ is evaluated, which can be written as

$$
\frac{1}{2} \mathcal{E}\left\{\log \operatorname{det}\left[\mathbf{D}^{H} \mathbf{D} \mathbf{C}^{-1}\right]\right\}=\mathcal{E}\left\{\frac{1}{2} \log \beta_{0}^{2}+\frac{1}{2} \log \frac{\left|h_{R D}\right|^{2} \beta_{1}^{2}}{\left|h_{R D}\right|^{2}+\beta_{1}^{2}}\right\} .
$$

Note $\beta_{0}$ is Chi-square distributed with 4 degree of freedom, and hence we can have $\mathcal{E}\left\{\frac{1}{2} \log \beta_{0}^{2}\right\}=\frac{1}{2} \psi(2) \log e$. Define $z=\frac{\left|h_{R D}\right|^{2} \beta_{1}^{2}}{\left|h_{R D}\right|^{2}+\beta_{1}^{2}}$ and its CDF has been developed in the previous section

$$
P(z)=\left\{1-2 z^{2} e^{-2 z}\left[\mathbf{K}_{1}(2 z)+\mathbf{K}_{2}(2 z)\right]\right\}^{L} .
$$

By using the factor $\mathbf{K}_{1}(2 z) \geq 0$ for $z \geq 0$, this CDF can be upper bounded as

$$
P(z) \leq\left\{1-2 z^{2} e^{-2 z} \mathbf{K}_{2}(2 z)\right\}^{L} .
$$

Recall that the Bessel function can be expressed as the following integral

$$
\mathbf{K}_{2}(z)=\frac{z^{2} \Gamma\left(\frac{1}{2}\right)}{2^{2} \Gamma\left(\frac{5}{2}\right)} \int_{1}^{\infty} e^{-z t}\left(t^{2}-1\right)^{\frac{3}{2}} d t
$$

which can be lower bounded as

$$
\mathbf{K}_{2}(z) \geq \frac{z^{2}}{3 ! !} \int_{1}^{\infty} e^{-z t}(t-1)^{3} d t=2 \frac{e^{-z}}{z^{2}},
$$

where the inequality follows the assumption $t \geq 1$. Applying this simplified form to (21), the CDF of $z$ can be finally upper bounded as $P(z) \leq\left\{1-e^{-4 z}\right\}^{L}$.

To obtain the lower bound, observe that the Bessel function can be expressed as the integral form

$$
\mathbf{K}_{2}(z)=\frac{z^{2}}{8} \int_{0}^{\infty} \frac{e^{-t-\frac{z^{2}}{4 t}}}{t^{3}} d t
$$

which provides the following inequality

$$
\mathbf{K}_{2}(z) \leq \frac{z^{2}}{8} \int_{0}^{\infty} \frac{e^{-\frac{z^{2}}{4 t}}}{t^{3}} d t=\frac{2}{z^{2}}
$$

where the inequality follows the assumption $e^{-t} \geq 1$ for $t \geq 0$. Following the similar step, the bessel function with first order can be upper bounded as $\mathbf{K}_{2}(z) \leq \frac{1}{z}$. By using the bounds of the bessel functions, the CDF can be bounded as

$$
\left\{1-e^{-4 z}\right\}^{L} \geq P(z) \geq\left\{1-e^{-2 z}(1+z)\right\}^{L} .
$$


An observation is that the lower bound of the CDF is still not helpful to obtain the explicit expression. A simple inequality $1+z \leq e^{z}$ holds for $z \geq 0$, which can further simplify the bounds of the CDF as

$$
\left\{1-e^{-4 z}\right\}^{L} \geq P(z) \geq\left\{1-e^{-z}\right\}^{L},
$$

since $1+z \leq e^{z}$ for $z \geq 0$. As can be seen from (27), both the upper and lower bounds share the same structure, which will simplify the following development. Furthermore, both two bounds resembles the CDF of the largest value among $L$ i.i.d. exponentially distributed variables.

Following the similar steps in [7], the expectation of the variable $\log z$ can be bounded as

$$
f(4) \leq \mathcal{E}\{\log z\} \leq f(1)
$$

where $f(\cdot)$ is defined as

$$
f(\lambda)=\int_{0}^{\infty} \lambda L e^{-\lambda z}\left(1-e^{-\lambda z}\right)^{L-1} \log z d z .
$$

By applying binomial expansion, we can have

$$
f(\lambda)=\log e \sum_{k=1}^{L} C_{L}^{k}(-1)^{k}[\mathbf{C}+\ln \lambda k] .
$$

Utilizing the fact that $\sum_{k=1}^{L} C_{L}^{k}(-1)^{k}=-1$, the expectation of $\log z$ can be bounded as

$$
\begin{array}{r}
-\mathbf{C} \log e+\sum_{k=1}^{L} C_{L}^{k}(-1)^{k} \log 4 k \leq \mathcal{E}\{\log z\} \\
\leq-\mathbf{C} \log e+\sum_{k=1}^{L} C_{L}^{k}(-1)^{k} \log k
\end{array}
$$

And evidently the difference of the upper and lower bound is $f(1)-f(4)=2$.

The expectation of the variable $\log \operatorname{det}\left\{\mathbf{H} \mathbf{H}^{H}\right\}$ can be obtained in an explicit expression as the following. Different to regular random matrices, each row of $\mathbf{H}$ is normalized, and furthermore the elements for the second row is no longer Gaussian distributed because of relay selection. The relay $R$ is chosen since it has the largest value of $\frac{\left|h_{R_{l} D}\right|^{2} \beta_{l}^{2}}{\left|h_{R_{l} D}\right|^{2}+\beta_{l}^{2}}$ among the $L$ relay candidates. This implicit structure of $\mathbf{H}$ has the impact on its distribution and hence complicates the calculation.

An important observation is that the relay selection criterion $\frac{\left|h_{R_{l} D}\right|^{2} \beta_{l}^{2}}{\left|h_{R_{l} D}\right|^{2}+\beta_{l}^{2}}$ is only a function of the norm of each row, and not directly related with each element. Since each row of $\mathbf{H}$ has been normalized, the effect of relay selection on the distribution has been removed. Hence construct two $2 \times 1$ vectors, $\mathbf{v}_{1}, \mathbf{v}_{2}$, whose elements are i.i.d. Raleigh distributed. The density function of the determinant $\operatorname{det}\left[\mathbf{H} \mathbf{H}^{H}\right]$ is the same as the following determinant

$$
\operatorname{det}\left[\overline{\mathbf{D}} \overline{\mathbf{H}} \overline{\mathbf{H}}^{H}\right]
$$

where $\overline{\mathbf{H}}=\left[\begin{array}{ll}\mathbf{v}_{1}^{H} & \mathbf{v}_{2}^{H}\end{array}\right], \overline{\mathbf{D}}=\operatorname{diag}\left\{\alpha_{1}, \alpha_{2}\right\}$ and $\alpha_{n}=$ $1 / \mathbf{v}_{n} \mathbf{v}_{n}^{H}$. Note that $\overline{\mathbf{H}} \overline{\mathbf{H}}^{H}$ is the classical Complex Random Wishart Matrix whose determinant has the following distribution [10]

$$
\operatorname{det}\left\{\overline{\mathbf{H}} \overline{\mathbf{H}}^{H}\right\} \sim \prod_{i=1}^{2} u_{i}
$$

where $u_{i}$ are independent to each other and $u_{i} \sim \chi_{2 i}^{2}$.

By using such a result, the addressed expectation can be evaluated as

$$
\begin{aligned}
\mathcal{E}\left\{\log \operatorname{det}\left[\mathbf{H} \mathbf{H}^{H}\right]\right\} & =\mathcal{E}\left\{\log \operatorname{det}\left[\overline{\mathbf{D}} \overline{\mathbf{H}} \overline{\mathbf{H}}^{H}\right]\right\} \\
& =\mathcal{E}\left\{\log \prod_{i=1}^{2} \alpha_{i}\right\}+\mathcal{E}\left\{\log \operatorname{det}\left[\overline{\mathbf{H}} \overline{\mathbf{H}}^{H}\right]\right\}
\end{aligned}
$$

Note that $\alpha_{i}$ is Chi-square distributed with 4 degree of freedom. Together using the distribution in (32), we can have

$$
\begin{gathered}
\mathcal{E}\left\{\log \operatorname{det}\left[\mathbf{H H}^{H}\right]\right\}=-\int_{0}^{\infty} z \log z e^{-z} d z \\
+\int_{0}^{\infty} \log x e^{-x} d x=\log e[\psi(1)-\psi(2)] .
\end{gathered}
$$

Note that $\psi=-$ C. Combining (31) and (34), the proof is completed.

The capacity difference between the proposed protocol and direct transmission can be bounded as

$$
\frac{1}{2}\left(\sum_{k=1}^{L} C_{L}^{k}(-1)^{k} \log 4 k\right) \leq \mathcal{E}-\mathcal{E}_{D} \leq \frac{1}{2}\left(\sum_{k=1}^{L} C_{L}^{k}(-1)^{k} \log k\right)
$$

Provided that there are enough number of relays, the lower bound of the difference can be positive, and hence the ergodic capacity larger than that of direct transmission can be achieved by the proposed scheme.

\section{NuMERiCAL RESUlts}

In this section, the performance of the proposed transmission protocol is evaluated by using Monte-Carlo simulations. The performance of direct transmission and the best-relay scheme [2] is also shown for comparison. Only $M=2$ sources are involved in user cooperation and all channels are assumed i.i.d. Raleigh fading. In Fig. 2, the outage probability is shown as a function of SNR, where the number of relays is set as $L=2$ and the targeted per user data rate is set as $R=4 \mathrm{bits} / \mathrm{s} / \mathrm{Hz}$. As can be seen from the figure, for practical SNR range, the proposed scheme can achieve smaller outage probability than two comparable schemes. Furthermore, the use of the distributed relay selection strategy does not cause large performance penalty compared with the optimal one. In Fig. 3, the ergodic capacity achieved by the four schemes is shown as a function of SNR, where the number of the relays is set as $L=2$ and $L=10$. Consistent to our analytical results, both the two proposed network coded cooperative schemes can achieve larger ergodic capacity than direct transmission, whereas the existing cooperative scheme can only realize a faction of the capacity achieved by direct transmission. The reason for such significant gain of ergodic capacity is that the use of network coding ensures the suppression of bandwidth resource wasted by relay transmission. In specific, one single relay transmission can serve more than one source nodes simultaneously, and hence the spectral efficiency of cooperative diversity can be improved substantially. 


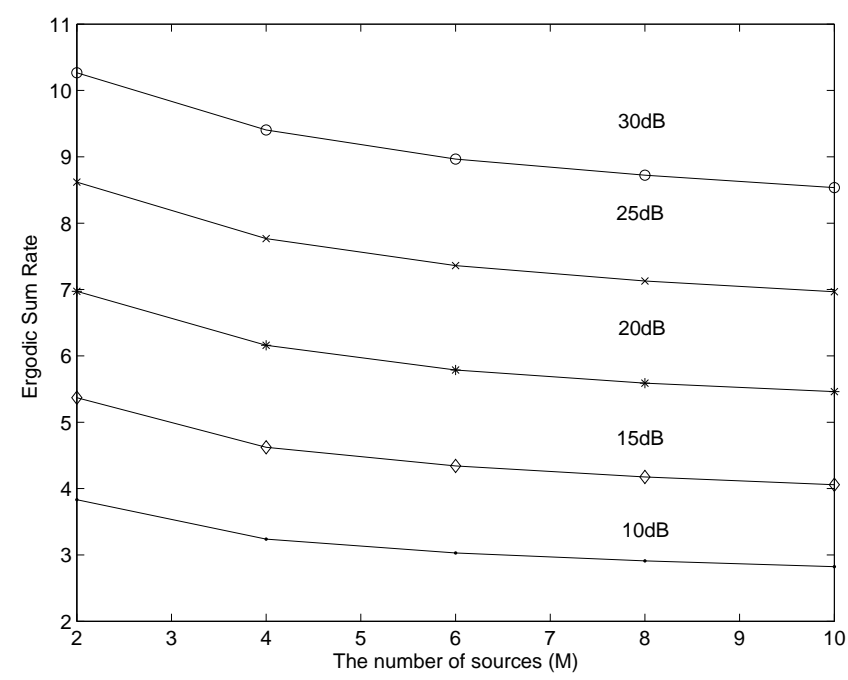

Fig. 1. The ergodic sum rate vs the number of sources participating cooperation. The number of relays is $L=10$.

\section{CONCLUSION}

In this paper, we provided detailed analytical performance evaluation for the proposed network coded cooperative multiple access channels. With the help of the distributed relay selection strategy, the explicit expressions of the outage probability and ergodic capacity can be obtained. Both analytical and numerical results demonstrated that the proposed cooperative protocol can achieve larger ergodic capacity while maintaining the property of full diversity gain.

\section{REFERENCES}

[1] J. N. Laneman, D. N. C. Tse, and G. W. Wornell, "Cooperative diversity in wireless networks: Efficient protocols and outage behavior," IEEE Trans. Information Theory, vol. 50, pp. 3062-3080, Dec. 2004.

[2] A. Bletsas, A. Khisti, D. P. Reed, and A. Lippman, "A simple cooperative diversity method based on network path selection," IEEE Journal on Select. Areas in Comm., vol. 24, pp. 659-672, Mar. 2006.

[3] K. Azarian, H. E. Gamal, and P. Schniter, "On the achievable diversitymultiplexing tradeoff in half-duplex cooperative channels," IEEE Trans. Information Theory, vol. 51, pp. 4152-4172, Nov. 2005.

[4] R. Ahlswede, N. Cai, S. R. Li, and R. W. Yeung, "Network information flow," IEEE Trans. Information Theory, vol. 46, pp. 1204-1217, Jul. 2000.

[5] S. Zhang, S. Liew, and P. Lam, "Physical layer network coding," in Proc. 12th Annual International Conference on Mobile Computing and Networking (ACM MobiCom 2006), Sept. 2006.

[6] Y. Chen, S. Kishore, and J. Li, "Wireless diversity through network coding," in Proceeding of IEEE Wireless Communications and Networking Conference (WCNC), Mar. 2006, pp. 1681 - 1686.

[7] Z. Ding, K. K. Leung, D. L. Goeckel, and D. Towsley, "On the study of network coding with diversity," IEEE Trans. on Wireless Communications, (to appear in 2008).

[8] D. N. C. Tse, P. Viswanath, and L. Zheng, "Diversity-multiplexing tradeoff in multiple-access channels," IEEE Trans. Information Theory, vol. 50, pp. 1859-1874, Sept. 2004.

[9] Z. Ding, T. Ratnarajah, and C. Cowan, "On the diversity-multiplexing tradeoff for wireless cooperative multiple access systems," IEEE Trans. Signal Processing, pp. 4627-4638, Sept. 2007.

[10] N. R. Goodman, "The distribution of the determinant of a complex wishart distributed matrix," The Annals of Mathematical Statistics, vol. 34, pp. 178-180, Mar. 1963.

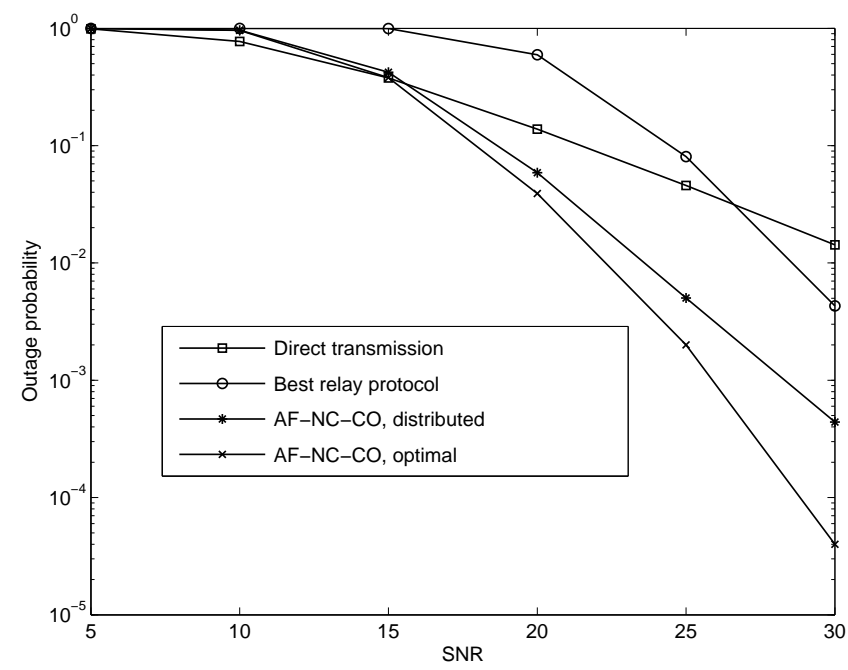

Fig. 2. Outage probability vs SNR. The data rate is set as $R=4 \mathrm{bits} / \mathrm{Hz} / \mathrm{s}$. The number of relays is $L=2$.

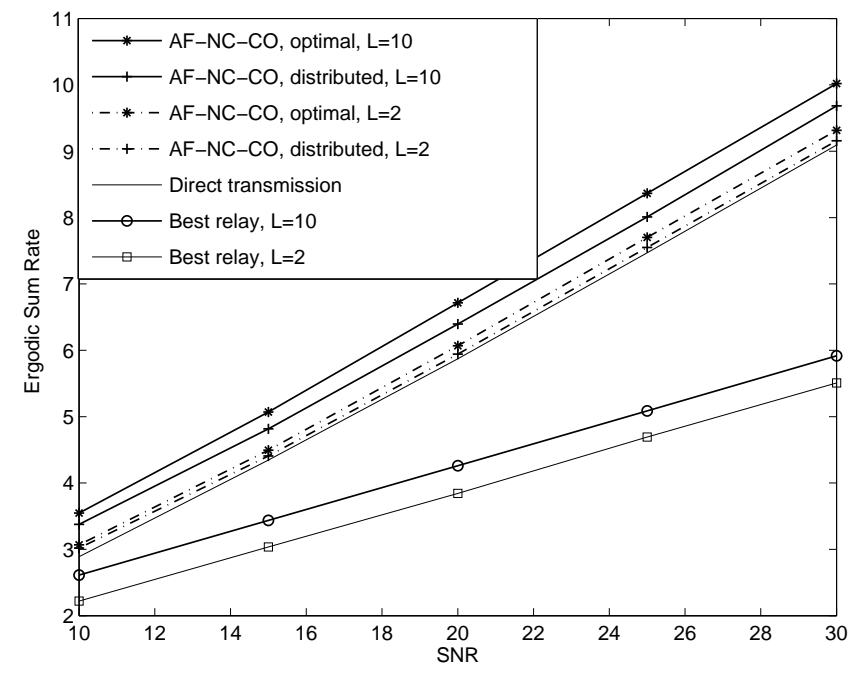

Fig. 3. Ergodic sum rate vs SNR. The number of the source nodes is $M=2$.

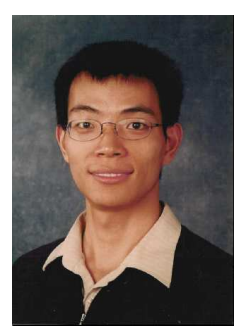

Zhiguo Ding (S'03-M'05) received his B.Eng in Electrical Engineering from the Beijing University of Posts and Telecommunications in 2000, and the Ph.D degree in Electrical Engineering from Imperial College London in 2005.

From 2000 to 2002, he was a soft engineer in Datang Telecom Inc. and Vimicro Corporation, Beijing, P.R.China. From Jul. 2005 to Sept. 2007, he was with Queen's University Belfast and Imperial College as a postdoctoral researcher. Since Oct. 2008, he has been with the Department of Communication Systems, Lancaster University, as a Lecturer. His research interests are cross-layer optimization, cooperative diversity, statistical signal processing and information theory. 


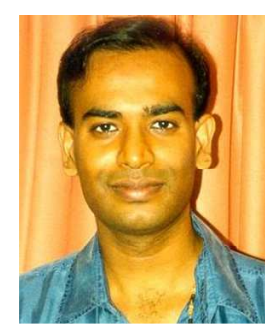

T. Ratnarajah (S'94-A'96-M'05-SM'05) holds B. Eng. (Hons), M.Sc. and Ph.D. degrees. Since 1993 he has held various research positions at University of Ottawa, Nortel Networks, McMaster University and Imperial College. He is currently a Principal Research Engineer at the Institute for Electronics, Communications and Information Technologies (ECIT) at Queen's University Belfast, Belfast, UK. His research interests include random matrices theory, information theoretic aspects of MIMO channels and ad hoc networks, wireless communications, signal processing for communication, statistical signal processing, biomedical signal processing and quantum information theory. He has published over 50 publications in these areas, including four US patents.

Dr T. Ratnarajah is a senior member of IEEE, member of American Mathematical Society and Information Theory Society.

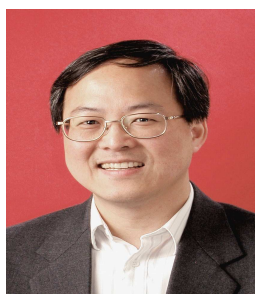

Kin K. Leung received his B.S. degree (with firstclass honors) from the Chinese University of Hong Kong in 1980, and his M.S. and Ph.D. degrees in computer science from University of California, Los Angeles, in 1982 and 1985, respectively.

He started his career at AT\&T Bell Labs in 1986 and worked at its successor companies, AT\&T Labs and Bell Labs of Lucent Technologies, until 2004. Since then, he has been the Tanaka Chair Professor in Internet Technology at Imperial College in London. His research interests include network resource allocation, MAC protocol, TCP/IP protocol, distributed optimization algorithms, mobility management, network architecture, real-time applications and teletraffic issues for broadband wireless networks, wireless sensor and adhoc networks. He is also interested in a wide variety of wireless technologies, including IEEE 802.11, 802.16, and 3G and future generation cellular networks.

He received the Distinguished Member of Technical Staff Award from AT\&T Bell Labs in 1994, and was a co-recipient of the 1997 Lanchester Prize Honorable Mention Award. He was elected in 2001 as an IEEE Fellow for his contributions to performance analysis, protocol design, and control algorithms for communications networks. He receives the Royal Society Wolfson Research Merits Award from 2004 to 2009. He has published widely and acquired patents in many areas of communication networks. He has actively served on many conference committees, including as the committee co-chair for the Multiaccess, Mobility and Teletraffic for Wireless Communications (MMT'98), the Wireless Networking Theory Symposium of IEEE ICC 2002 and the First Annual Conference of International Technology Alliance (ITA) 2007 (sponsored by the U.S. Army and U.K. Ministry of Defence). He was a guest editor for the IEEE Journal on Selected Areas in Communications (JSAC), IEEE Wireless Communications and the MONET journal, and as an editor for the JSAC: Wireless Series. Currently, he is an editor for the IEEE Transactions on Communications, the Transactions on Wireless Communications and International Journal on Sensor Networks. 